\title{
MINIMISATION PROBLEMS FOR IMPLICIT FUNCTIONALS DEFINED BY DIFFERENTIAL EQUATIONS OF LIVER KINETICS
}

\author{
L. BASS, A. J. BRACKEN and R. VYBORNY ${ }^{1}$
}

(Received 21 October 1982; revised 15 February 1983)

\begin{abstract}
A substance carried convectively through the liver by the blood undergoes two successive enzymatic transformations. The resulting concentrations of the three forms of the substance are determined, as functions of position along the blood flow in the steady state, by coupled ordinary differential equations of the first order on a finite interval. The densities along the blood flow of the activities of the two (immobile) transforming enzymes are described by two non-negative and normalised functions of position.

The problem, suggested by recent experimental results, is to choose these two functions so as to minimise the concentration of the once-transformed (intermediate) form of the substance at one boundary (the liver outlet). That minimisation is particularly significant biologically when the intermediate form is toxic and the second transformation renders it harmless. In this problem of optimal control (exerted perhaps by natural selection), the classical approach through Euler's equations is inapplicable because of the constraints on the two density functions. Moreover, $w$ ten the enzyme kinetics and hence the differential equations are non-linear, the functional to be minimised is not obtainable explicitly. Instead it appears, after some manipulation of the coupled equations, as the terminal boundary value of the solution of a non-linear Volterra integral equation, which involves the two density functions (one explicitly and one implicitly) as control variables.

Appropriate existence, uniqueness and boundedness results are obtained for the solution of this integral equation, and the problem is then solved rigorously for one class of non-linearities (including saturation kinetics). Some unanswered questions are posed for another class (including substrate-inhibition kinetics).
\end{abstract}

\section{Introduction}

Several physiological functions of the liver involve blood-borne substances which enter liver cells and there undergo single or successive transformations as a result of activities of cellular enzymes. In this context the substances are called

\footnotetext{
1 Department of Mathematics, University of Queensland, St. Lucia, 4067, Queensland.

(c) Copyright Australian Mathematical Society 1984, Serial-fee code 0334-2700/84
} 
substrates of the enzymes. The concentrations of the substrates can be monitored in the blood at the inlet and at the outlet of the liver, and they can be varied experimentally at will at the inlet. What conclusions can be drawn about the spatial distributions of the enzyme activities over the intact liver, from the way the liver transforms the influxes of substrates into outfluxes? In particular: would all liver cells show the same enzyme activities if the same amounts of substrates were presented to them by the blood? It was long believed that they would [8], that is, that liver cells are functionally homogeneous.

However, recent analyses [2,3] of experimental data [10] concerning particular substrates suggest that, quite to the contrary, qualitatively different enzyme activities take place in distinct spatial zones arranged along the direction of blood flow. This arrangement appears to be such as to minimise the outflux concentration of a metabolite substrate made in liver cells from a precursor substrate. When the metabolite is toxic, such minimisation amounts to optimal control of the detoxification of the liver as a biochemical reactor (see Section 6).

When this hypothesis is to be examined mathematically, allowance must be made from the outset for the gradients of substrate concentrations that develop between the inlet and the outlet as a result of the interplay between the enzyme kinetics in each liver cell, and the unidirectional blood flow through the liver. The problem has so far been studied only for linear (first order) enzyme kinetics [2,3], and even then somewhat heuristically. Such kinetics are valid at low substrate concentrations, whereas physiologically important forms of cellular enzyme kinetics are often non-linear at higher concentrations [7].

In the present work we therefore generalise this minimisation problem to include realistic types of non-linear cellular enzyme kinetics, we solve it rigorously for one class of such kinetics, including linear kinetics, and we pose some unsolved problems for another class. In general, the functional to be minimised in each case (representing the outflux concentration of metabolite) cannot be written down explicitly, but is defined implicitly by a pair of coupled non-linear differential or integral equations (ultimately reduced to one). These involve two density functions which describe the fixed longitudinal distributions of the two relevant enzyme types in the liver, and which are essentially the "control variables" in the problem. That these functions must be nonnegative and normalised makes it impossible to apply the classical methods of the calculus of variations. The problem of existence of a minimising pair is further complicated by the fact that the minimum is sought over a set which is not compact, and not even weakly compact.

Because of the novelty of the mathematical problem, we state it at the outset unencumbered by its unfamiliar biological background. Let $\lambda$ and $L$ be given positive constants, and let $\beta$ be a given real-valued function on $[0, \lambda]$, Lipschitz and non-negative. Consider all pairs of real-valued functions $\{\Phi, g\}$ on $[0, L]$, 
with $\Phi$ absolutely continuous and nondecreasing from $\Phi(0)=0$ to $\Phi(L)=\lambda$; and with $g$ non-negative and Lebesgue integrable, and such that

$$
\int_{0}^{L} g(x) d x=1 .
$$

Then, for each such pair and for $0 \leqslant x \leqslant L$, consider the function $M(x)$ defined by the integral equation

$$
M(x)=\Phi(x)-\int_{0}^{x} g(s) \beta(M(s)) d s .
$$

The problem is to find which pair (or pairs) $\{\Phi, g\}$ minimise the value $M(L)$.

Before this problem can sensibly be tackled, certain preliminary questions arise, in particular concerning the existence, uniqueness and boundedness of the solution of equation (1.2) which is in general non-linear. The proofs of the appropriate theorems, which are stated in Section 4, are presented in Appendices A and B. (It is not sufficient to refer to standard theorems which provide only local results for non-linear equations, or global results for linear equations.)

Following an introductory examination in Section 3 of the simplest case, where $\beta(M)=m M$ with $m$ a positive constant, we solve the problem completely in Section 4 in the more general case that $\beta^{\prime}>0$ on $(0, \lambda), \beta$ being continuously differentiable on $[0, \lambda]$. Certain unanswered questions are raised in Section 5 concerning cases where $\beta$ does not increase monotonically on $[0, \lambda]$. Partial results on the existence of minimising pairs $\{\Phi, g\}$ in these cases are provided in Appendix C.

The physiological and biochemical modelling leading to this mathematical problem is concentrated in Section 2. Only a few interpretative biological comments seemed essential in Sections 3-5, and these are enclosed in square brackets in order not to impede the flow of the mathematical argument.

\section{Physiological background and formulation}

The contact between blood and liver cells is optimised in the healthy liver by the manifolding of the blood flow through many parallel capillaries, called liver sinusoids, which are lined with monolayers of liver cells and are just wide enough to pass blood cells (the sinusoidal flows reunite at the outlet into the liver vein). These special circumstances lead to considerable simplifications of the modelling: we refer to previous work $([1,5]$, and references therein) for their detailed justification. Substrate diffusion along the sinusoid is negligible compared with convective transport. Blood flow defines a convective transit time through each sinusoid. On that time-scale, substrate equilibration across each sinusoidal crosssection (including, for substrates considered here, transport into and out of liver 
cells) is so rapid that the activities of the cellular enzymes can be described in terms of volume sinks and sources, rather than by boundary conditions on the walls of the sinusoids.

We consider steady-state enzymatic transformations of a substrate in two consecutive steps. A precursor of concentration $P \geqslant 0$ is infused steadily through the inlet and is transformed by a cellular enzyme into a metabolite of concentration $M \geqslant 0$, which is in turn transformed by another cellular enzyme into its conjugate of concentration $\hat{M} \geqslant 0$. The precursor, the metabolite and its conjugate are exchanged steadily between the liver cells and the ambient blood, which sweeps all three species towards the outlet.

In a single sinusoid, we put the $x$-axis along the blood flow, with inlet at $x=0$ and outlet at $x=L$. The rate of blood flow through the sinusoid is denoted by $F$, and since the flow is practically incompressible, the value of $F$ is independent of $x$ even if the cross-sectional area of the sinusoid varies with $x$. Blood cells filing through the sinusoid hinder the establishment of radial velocity distributions (such as the Poiseuille distribution found in larger vessels), so that the use of only the total rate $F$ of sinusoidal flow suffices for describing effects of convection in the present context.

Suppose that the rate of transformation of the precursor to the metabolite at each relevant enzyme molecule is given by a non-negative function $a(P)$ of the local concentration $P$ of precursor. There is such a large number $N_{p}$ of these enzyme molecules that we may think of a continuously varying density of them along the sinusoid, the fraction of them between the cross-sections at $x$ and $x+\delta x$ being $f(x) \delta x$, with

$$
f(x) \geqslant 0, \quad \int_{0}^{L} f(x) d x=1 .
$$

(Actually, the condition that $f$ be continuous is unnecessarily stringent, and we shall shortly relax it.) The totality of such enzyme molecules positioned along the sinusoid would give a transformation rate $N_{P} a(P)$ if they all met the same precursor concentration $P$. In fact, $P$ varies with $x$ from $P(0)$ at the inlet to $P(L)$ $[<P(0)]$ at the outlet, and the transformation rate between $x$ and $x+\delta x$ is therefore given by $N_{p} f(x) a(P(x)) \delta x$. The steady-state continuity equation for $P(x)$ is then

$$
F P^{\prime}(x)=-N_{P} f(x) a(P) .
$$

The decrease in $P$ between $x$ and $x+\delta x$ provides the source of $M$, which is in turn decreased by transformation to $\hat{M}$ by a second type of enzyme. Each molecule of this second enzyme is assumed to act at a rate given by a certain non-negative function $b(M)$, and the large number $N_{M}$ of them is assumed to be distributed over the sinusoid in accordance with a second density function $g(x)$. 
Then, in the steady state,

$$
F M^{\prime}(x)=N_{P} f(x) a(P)-N_{M} g(x) b(M),
$$

where

$$
g(x) \geqslant 0, \quad \int_{0}^{L} g(x) d x=1 .
$$

Similarly, the decrease in $M$ between $x$ and $x+\delta x$ provides the scurce of $A \hat{A}$; thus

$$
F \hat{M}^{\prime}(x)=N_{M} g(x) b(M) .
$$

Measurements on the liver provide information about $F$ and the functions $N_{P} a(P), N_{M} b(M)$ as well as the boundary values of $P, M, \hat{M}$ at $x=0$ and $x=L$ [3]. We treat the functions $\alpha$ and $\beta$, defined by

$$
\alpha(P)=N_{P} a(P) / F, \quad \beta(M)=N_{M} b(M) / F,
$$

as given in what follows, and we refer to them as kinetic functions. They are typically continuous on $[0, \infty)$ and positive on $(0, \infty)$, with

$$
\alpha(0)=0=\beta(0)
$$

and each typically has a finite positive derivative (from the right) at $P=0$ or $M=0$, whichever is appropriate. This last condition reflects the circumstance that linear (first-order) kinetics is necessarily approached at sufficiently low substrate concentrations. These properties will be assumed in what follows. Other conditions (on $\beta$ in particular) will be described as needed.

One simple possibility is

$$
\alpha(P)=p P, \quad \beta(M)=m M,
$$

with $p, m$ positive constants. Then one has exactly linear kinetics at all substrate concentrations for each type of enzyme. But there are other important possibilities, such as [7]

$$
\begin{aligned}
& \text { saturation (Michaelis-Menten) kinetics: } \beta(M)=\frac{m M}{1+M / K_{1}}, \\
& \text { substrate-inhibition kinetics: } \beta(M)=\frac{m M}{1+M / K_{1}+M^{2} / K_{1} K_{2}},
\end{aligned}
$$

where $m, K_{1}, K_{2}$ are positive constants. Similar possibilities exist for $\alpha$, and the kinetic functions $\alpha$ and $\beta$ need not be the same for the two enzyme types.

In terms of $\alpha$ and $\beta$, equations $(2.2,2.3,2.5)$ become

$$
\begin{gathered}
P^{\prime}(x)=-f(x) \alpha(P), \\
M^{\prime}(x)=f(x) \alpha(P)-g(x) \beta(M), \\
\hat{M}^{\prime}(x)=g(x) \beta(M) .
\end{gathered}
$$


We take

$$
M(0)=0, \quad P(0)=P_{0}>0 .
$$

Then all metabolite appearing at the outlet from the sinusoid is produced in the sinusoid from the precursor. We therefore expect, and shall prove below, that for all $x \in[0, L]$,

$$
P(x) \leqslant P_{0}, \quad 0 \leqslant M(x) \leqslant \lambda
$$

where

$$
\lambda=P_{0}-P(L) \text {. }
$$

The boundary values of $\hat{M}$, and indeed the differential equation (2.9c), do not concern us here. Our main problem, for given $\alpha$ and $\beta$, and given $P_{0}$, is to minimise the value of $M(L)$ by suitably choosing the density functions $f$ and $g$ subject to the constraints $(2.1,2.4)$. The value of $M(L)$ may be regarded as a functional of $f$ and $g$ which is determined implicitly by the pair of equations $(2.9 \mathrm{a}, \mathrm{b})$, with boundary conditions $(2.10)$. We are mainly interested in cases where the minimising $f$ and $g$ do not in fact depend upon $P_{0}$; this point will be discussed below. (See Section 5 in particular.)

With regard to the smoothness of the functions $f$ and $g$, it is most natural to require only their (Lebesgue) integrability. Accordingly, the differential equations, in particular $(2.9 \mathrm{a}, \mathrm{b})$, should be interpreted in Carathéodory's sense [6]; equivalently, we work instead with the corresponding Volterra integral equations

$$
\begin{gathered}
P(x)=P_{0}-\int_{0}^{x} f(s) \alpha(P(s)) d s, \\
M(x)=\int_{0}^{x}[f(s) \alpha(P(s))-g(s) \beta(M(s))] d s .
\end{gathered}
$$

It can be seen from the separability of (2.9a) (and the properties assumed of $\alpha$ ) that the solution of (2.13a) is the absolutely continuous, non-increasing function $P(x)$ defined by

$$
\int_{P_{0}}^{P(x)} \frac{d P}{\alpha(P)}=-\int_{0}^{x} f(s) d s .
$$

The value of $P(L)$ is therefore independent of the choice of $f$ satisfying (2.1), and is defined by

$$
\int_{P_{0}}^{P(L)} \frac{d P}{\alpha(P)}=-1
$$

Then $\lambda$ as in (2.12) is also independent of $f$, and $0<\lambda<P_{0}$.

For each $f$, we can define

$$
\Phi(x)=P_{0}-P(x)
$$


and then $\Phi$ is absolutely continuous and non-decreasing on $[0, L]$, with

$$
\Phi(0)=0, \quad \Phi(L)=\lambda .
$$

Conversely, given an absolutely continuous and non-decreasing $\Phi$ satisfying (2.17), we can recover the corresponding $f$ modulo a set of measure zero, by defining $P(x)$ via (2.16) and then using (2.9a).

Now (2.13b) can be written as

$$
M(x)=\Phi(x)-\int_{0}^{x} g(s) \beta(M(s)) d s .
$$

We thus arrive at the mathematical problem stated in the introduction. We seek a pair (or pairs) $\{\Phi, g\}$, with $\Phi$ an absolutely continuous and non-decreasing function on $[0, L]$ satisfying (2.17), and $g$ an integrable function on $[0, L]$ satisfying (2.4), which together minimise the value at $x=L$ of the solution to (2.18). [In Section 4 we prove, under suitable conditions on $\beta$, the existence and uniqueness of the solution of (2.18) for each $\lambda$ and each candidate pair $\{\Phi, g\}$. Moreover, we show that this solution is (absolutely) continuous on $[0, L]$ and satisfies (2:11b).]

The following section contains an outline analysis of those cases where the conjugation kinetics, associated with the kinetic function $\beta$, is linear. The metabolisation kinetics, associated with $\alpha$, is left arbitrary to within the constraints imposed above. In these cases one can obtain an explicit expression for the functional to be minimised, and although the analysis will be superseded by the rigorous and more general treatment to be given in Section 4 , its presentation will elucidate the nature of the problem and its solution.

\section{The case of a linear kinetic function $\beta(M)$}

Here we have $\beta(M)=m M$, and the solution of $(2.18)$ is seen to be

$$
M(x)=e^{-\Psi(x)} \int_{0}^{x} e^{\Psi(s)} \Phi^{\prime}(s) d s,
$$

where

$$
\Psi(x)=m \int_{0}^{x} g(s) d s .
$$

Note that the conditions (2.4) on $g$ define $\Psi$ as an absolutely continuous, non-decreasing function on $[0, L]$, with

$$
\Psi(0)=0, \quad \Psi(L)=m .
$$


Conversely, every absolutely continuous non-decreasing $\Psi$ satisfying (3.3), defines almost everywhere a $g$ of the required type through (3.2). We see from (3.1) and (3.3b) that

$$
M(L)=e^{-m} \int_{0}^{L} e^{\Psi(x)} \Phi^{\prime}(x) d x .
$$

Our problem in these cases therefore reduces to the following: find two absolutely continuous, non-decreasing functions $\Phi$ and $\Psi$ on $[0, L]$, satisfying (2.17) and (3.3), which minimise the value of the functional

$$
A=\int_{0}^{L} e^{\Psi(x)} \Phi^{\prime}(x) d x .
$$

We set

$$
X(x)=\Phi(x), \quad Y(x)=e^{\Psi(x)},
$$

and interpret $(X(x), Y(x))$ as Cartesian coordinates of a point on a curve, parametrized by $x$, and lying in the first quadrant of the $X Y$-plane (see Figure 1). Then we see that $A$ is simply the area bounded by this curve, the $X$-axis, and the lines $X=0$ and $X=\lambda$. We seek the curve which begins at $(0,1)$ and ends at $\left(\lambda, e^{m}\right)$, and which minimises this area, noting that since $\Phi$ and $\Psi$ are non-decreasing, the slope of this curve must be nowhere negative.

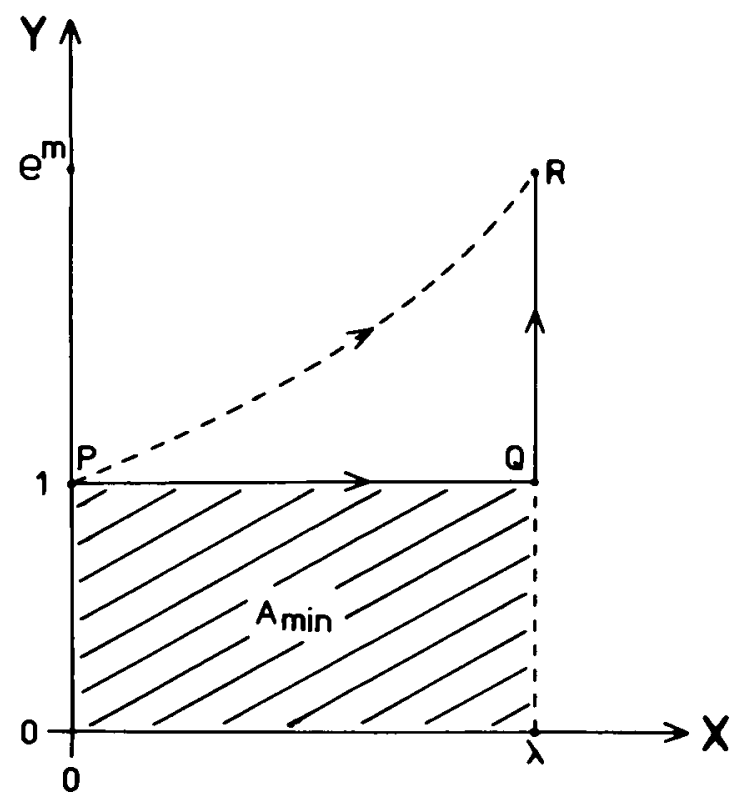

FIGURE 1. The two unknown functions of $x$ can be transformed to $X(x), Y(x)$ so that the path $P Q R$ in the $X Y$-plane minimises the integral of interest. The broken curve $P R$ corresponds to a classical view of liver cells, in a case of first-order metabolisation and conjugation kinetics, and is non-minimising. 
The required curve is obviously the one which runs from $(0,1)$ parallel to the $X$-axis to $(\lambda, 1)$, then parallel to the $Y$-axis to $\left(\lambda, e^{m}\right)$, and the corresponding minimum values of $A$ and $M(L)$ are

$$
A_{\min }=\lambda, \quad M(L)_{\min }=e^{-m} \lambda .
$$

We see that the corresponding $\Phi$ and $\Psi$ are by no means uniquely defined. Indeed, their dependence on $x$ is immaterial so long as the change in $\Phi$ from 0 to $\lambda$ is accomplished before the change in $\Psi$ commences, as $x$ runs from 0 to $L$. That is to say, it is required only that there exist a $\xi \in(0, L)$ such that

$$
\Phi(x)=\lambda, \quad x>\xi ; \quad \Psi(x)=0, \quad x<\xi .
$$

For such a $\Phi$ and $\Psi$, it follows from (2.16), (2.9a) and (3.2) that

$$
f(x)=0 \text { a.e., } \quad x>\xi ; \quad g(x)=0 \text { a.e., } \quad x<\xi .
$$

[Then effectively all the metabolising enzyme is upstream of all the conjugating enzyme. The interpretation of this result in terms of the enzyme kinetics seems intuitively clear: the outflux concentration of metabolite is minimised if all metabolite is created from the precursor upstream of the conjugating enzyme, so that none misses any chance of being conjugated. By contrast, the classical hypothesis of the functional homogeneity of liver cells [8] implies $f=g$ on $[0, L]$, defining a different curve between $(0,1)$ and $\left(\lambda, e^{m}\right)$ in the $X Y$-plane and, correspondingly, a larger value of $M(L)$.]

Consider for example the simplest case, when both kinetic functions are linear as in (2.7a,b). Equation (2.14) gives

$$
P(x)=P_{0} e^{-\chi(x)}
$$

where

$$
\chi(x)=p \int_{0}^{x} f(s) d s
$$

Then $P(L)=P_{0} e^{-p}$, so that

$$
\lambda=P_{0}\left(1-e^{-p}\right),
$$

and the smallest value possible for $M(L)$ is, from (3.7b),

$$
M(L)_{\min }=P_{0} e^{-m}\left(1-e^{-p}\right) .
$$

If on the other hand $f=g$ we have $\chi=p \Psi / m$, and then, from (2.16), (3.6) and (3.10), that on the corresponding curve in the $X Y$-plane

$$
Y=\left(1-X / P_{0}\right)^{-m / p} \text {. }
$$


For a typical $m$ and $p$, this is the broken curve in Figure 1. The corresponding value of $M(L)$ is

$$
M(L)= \begin{cases}\frac{p}{p-m}\left(e^{-m}-e^{-p}\right) P_{0} & \text { if } p \neq m, \\ m e^{-m} P_{0} & \text { if } p=m,\end{cases}
$$

which is greater than $M(L)_{\min }$ as in (3.13).

[It may be of interest to note here that when actual data from a case [10] with $\alpha(P)=p P, \beta(M)=m M$ (at low concentrations of $P$ and $M$ ) were evaluated statistically in terms of a simple overlap model of rectangular $f(x), g(x)$, the most probable overlap was zero, the median overlap was $17 \%$ and the odds against an overlap greater than $50 \%$ were more than $40: 1$. From the same data, (3.15) was refuted at the 0.004 level of statistical significance [3].]

\section{Rigorous solution for a monotonically increasing kinetic function $\beta(M)$}

The reader may now wonder whether it is not true that, whatever the form of the kinetic functions $\alpha(P)$ and $\beta(M)$, and whatever the value of $P_{0}$, the smallest value of $M(L)$ is always obtained when (essentially) all the metabolising enzyme is upstream of all the conjugating enzyme. Somewhat surprisingly perhaps, we shall show in Section 5 that this is not so. However, for given $\alpha(P)$, there is a class of kinetic functions $\beta(M)$ for which it is so, and it is for just this class that we now present a complete solution of the problem.

A given kinetic function $\beta(M)$ is in this class if it increases monotonically with $M$ for all values of $M$ that occur in the interval $0 \leqslant x \leqslant L$, whatever the concentration $P_{0}$ of precursor at entry into the sinusoid $(x=0)$. This happens independently of $\alpha(P)$ if $\beta(M)$ is monotonic for all positive $M$. It can happen also if $\beta(M)$ ceases to rise monotonically at some sufficiently high value of $M$, which however cannot be reached for any $P_{0}$ because of properties of $\alpha(P)$, such as (2.8b) [saturation of metabolisation]. The condition on $\beta$ for membership of this class depends on the form of $\alpha(P)$ as follows.

Consider the set of values of $\lambda\left[=P_{0}-P(L)\right.$, as in (2.12)] which are obtained as the value of $P_{0}$ runs over $(0, \infty)$. This set depends on $\alpha$ through $(2.15)$, which defines $P(L)$ and hence $\lambda$ for each $P_{0}$. Three types of set can occur (recall that $\alpha^{\prime}(0+)>0, \alpha(0)=0$, and $\alpha(P)>0$ for $\left.P>0\right)$ :
(i) $(0, \infty)$,
(ii) $(0, \bar{\lambda})$,
(iii) $(0, \bar{\lambda}]$,

with $\bar{\lambda}$ a positive constant. By examining the graph of the integrand in (2.15) in each case, one can see that examples of the three types are provided by (i) first-order kinetics (2.7a); (ii) Michaelis-Menten kinetics [cf. (2.8a)]; and (iii) 
substrate-inhibition kinetics [cf. (2.8b)]. In view of $(2.1 \mathrm{lb})$, and corresponding to (4.1), the requirement on $\beta$ for membership in the class under discussion is taken to be

$$
\beta^{\prime}(M)>0 \quad \text { for } \quad \text { (i) } M \in[0, \infty), \quad \text { (ii) } M \in[0, \bar{\lambda}), \quad \text { (iii) } M \in[0, \bar{\lambda}] \text {. }
$$

The case when $\beta$ is linear, as described in Section 3, is included here whatever the form of $\alpha$, as is the Michaelis-Menten form (2.8a), since in both cases we have $\beta^{\prime}(M)>0$ for all $M \geqslant 0$. But a substrate-inhibited kinetic function $\beta(M)$ as in (2.8b) is included only when the kinetic function $\alpha(P)$ leads to sets of $\lambda$-values of type (ii) or (iii) in (4.1), and then only for restricted values of the constants $K_{1}, K_{2}$ in (2.8b). [See equations (5.2).]

We now begin our exact analysis by establishing for (2.18), as promised in Section 2, that there exists a unique solution, that this solution is continuous, and that it satisfies $(2.11 \mathrm{~b}$ ), whatever the form of $\beta(M)$. For reasons which will become clear, we consider a more general equation than (2.18), involving a real parameter $\sigma$ :

$$
W(x, \sigma)=\theta(x, \sigma)-\int_{0}^{x} g(s) \beta(W(s, \sigma)) d s,
$$

in which $\theta, g$ and $\beta$ are supposed given, and $W$ is the unknown. In Appendix $A$ we present a proof, by the method of successive approximations, of:

THEOREM 1. Let $S=[0, L] \times[0,1]$. If

(i) $\theta: S \rightarrow \mathbf{R}$ is continuous;

(ii) $|\theta| \leqslant \lambda$ on $S, \lambda$ constant;

(iii) $g:[0, L] \rightarrow \mathbf{R}$ is integrable;

and either

(iva) $\beta: \mathbf{R} \rightarrow \mathbf{R}$ is Lipschitz, or

(ivb) $\beta$ is Lipschitz and non-negative on $[0, \lambda] ; g \geqslant 0 ; \theta \geqslant 0$; and for each $\sigma \in[0,1], \theta$ is a non-decreasing function of $x$ on $[0, L]$;

then (4.3) has a unique solution $W$, continuous on $S$.

Moreover, if (ivb) holds, then

$$
0 \leqslant W \leqslant \lambda
$$

on $S$. (Remark. If $\theta$ is absolutely continuous with respect to $x$ on $[0, L]$, then so is W.)

Taking $\theta(x, \sigma)=\sigma \Phi(x)$ and $M(x)=W(x, 1)$, we obtain the desired existence, uniqueness and boundedness results for (2.18), since conditions (i-iii) and (ivb) then hold. 
We return to consideration of (4.3), which we shall need to differentiate with respect to 0 . Proceeding formally, we get

$$
\frac{\partial W}{\partial \sigma}=\frac{\partial \theta}{\partial \sigma}-\int_{0}^{x} g(s) \beta^{\prime}(W) \frac{\partial W}{\partial \sigma} d s .
$$

Consider then the integral equation

$$
W_{1}(x, \sigma)=\frac{\partial \theta(x, \sigma)}{\partial \sigma}-\int_{0}^{x} g(s) \beta^{\prime}(W(s, \sigma)) W_{1}(s, \sigma) d s,
$$

in which $W_{1}$ is the unknown, and $W$ is determined by (4.3). Under suitable assumptions on $\beta^{\prime}$ and $\partial \theta / \partial \sigma$, equation (4.6) has a unique and continuous solution by Theorem 1 , and it is natural to expect that this solution $W_{1}$ is indeed $\partial W / \partial \sigma$. This is the content of:

THEOREM 2. If conditions (i-iii) of Theorem 1 hold, and in addition

(v) $\beta^{\prime}$ is continuous on $[0, \lambda] ; \beta \geqslant 0 ; g \geqslant 0$; and $\theta \geqslant 0$;

(vi) $\partial \theta / \partial \sigma$ is continuous on $S$;

then (4.6) has a unique solution $W_{1}$ on $S$, this solution is continuous on $S$, and it is the derivative with respect to $\sigma$ of the function $W$ satisfying (4.3).

For the more mathematically-minded reader, a proof is given in Appendix B.

Equipped with Theorem 2 we can prove the main results of this section.

THEOREM 3. Given a positive constant $\lambda$, let $\beta:[0, \lambda] \rightarrow \mathbf{R}$ be continuously differentiable, with $\beta \geqslant 0, \beta^{\prime} \geqslant 0$. Consider pairs $\{\Phi, g\}$ in which $\Phi:[0, L] \rightarrow \mathbf{R}$ is absolutely continuous and non-decreasing, and satisfies (2.17); and $g:[0, L] \rightarrow \mathbf{R}$ is integrable and satisfies (2.4). For each such pair, define $M$ by (2.18). Then a sufficient condition that the pair $\{\Phi, g\}$ leads to the minimum value of $M(L)$ is that $\{\Phi, g\}$ satisfies

Condition A. There exists a $\xi \in(0, L)$ such that

$$
\Phi(x)=\lambda, \quad x>\xi ; \quad g(x)=0 \text { a.e., } \quad x<\xi .
$$

If in addition, $\beta^{\prime}>0$ on $(0, \lambda)$, then this condition is also necessary.

Proof. For any pair $\{\Phi, g\}$, consider the equation

$$
W(x, \sigma)=\Phi(x)+\sigma(\lambda-\Phi(x))-\int_{0}^{x} g(s) \beta(W(s, \sigma)) d s,
$$

which is of the form (4.3). For $\sigma=0$, the solution of (4.8) becomes the solution of (2.18), and in particular

$$
W(L, 0)=M(L) .
$$


On the other hand, for $\sigma=1,(4.8)$ becomes

$$
\bar{M}(x)=\lambda-\int_{0}^{x} g(s) \beta(\bar{M}(s)) d s,
$$

where $\bar{M}(x)=W(x, 1)$. It is not difficult to solve (4.10) (by considering the associated differential equation, which is separable) and to see that while $\bar{M}(L)$ depends on $\lambda$ and $\beta$, it is independent of $\{\Phi, g\}$.

From (4.8) we have, with $W_{1}(x, \sigma)=\partial W(x, \sigma) / \partial \sigma$,

$$
W_{1}(x, \sigma)=\lambda-\Phi(x)-\int_{0}^{x} g(s) \beta^{\prime}(W(s, \sigma)) W_{1}(s, \sigma) d s .
$$

This is linear in $W_{1}$, and has the solution

$$
\begin{aligned}
W_{1}(x, \sigma)= & {\left[\lambda-\int_{0}^{x} \Phi^{\prime}(s) \exp \left(\int_{0}^{s} \beta^{\prime}(W(u, \sigma)) g(u) d u\right) d s\right] } \\
& \times \exp \left(-\int_{0}^{x} \beta^{\prime}(W(s, \sigma)) g(s) d s\right) .
\end{aligned}
$$

Then, noting

$$
\lambda=\int_{0}^{L} \Phi^{\prime}(s) d s
$$

we have

$$
\begin{aligned}
W_{1}(L, \sigma)= & \left\{\int_{0}^{L} \Phi^{\prime}(s)\left[1-\exp \left(\int_{0}^{s} \beta^{\prime}(W(u, \sigma)) g(u) d u\right)\right] d s\right\} \\
& \times \exp \left(-\int_{0}^{L} \beta^{\prime}(W(s, \sigma)) g(s) d s\right) .
\end{aligned}
$$

By Theorem $1, W(u, \sigma) \in[0, \lambda]$ in this equation, so $\beta^{\prime}(W(u, \sigma)) \geqslant 0$. Since $\Phi^{\prime} \geqslant 0$ almost everywhere, we have $W_{1}(L, \sigma) \leqslant 0$, that is

$$
\frac{\partial W(L, \sigma)}{\partial \sigma} \leqslant 0, \quad \sigma \in(0,1) \text {. }
$$

This implies

$$
W(L, 0) \geqslant W(L, 1)
$$

that is to say

$$
M(L) \geqslant \bar{M}(L) \text {. }
$$

Suppose $\{\Phi, g\}$ satisfies Condition A. Then for $\xi<x \leqslant L,(2.18)$ becomes

$$
M(x)=\lambda-\int_{\xi}^{x} g(s) \beta(M(s)) d s .
$$

However, for $\xi<x \leqslant L,(4.10)$ becomes

$$
\bar{M}(x)=\lambda-\int_{\xi}^{x} g(s) \beta(\bar{M}(s)) d s,
$$


and it is then clear that $M(x)=\bar{M}(x)$ for $\xi<x \leqslant L$, so that, in particular

$$
M(L)=\bar{M}(L)
$$

In view of (4.17) this proves the first part of the theorem.

Now suppose that $\beta^{\prime}>0$ on $(0, \lambda)$, and that the pair $\{\Phi, g\}$ minimises $M(L)$. Then $\partial W(L, \sigma) / \partial \sigma=W_{1}(L, \sigma)=0$ for $\sigma \in(0,1)$, and consequently

$$
\Phi^{\prime}(s)\left[1-\exp \int_{0}^{s} \beta^{\prime}(W(u, \sigma)) g(u) d u\right]=0
$$

almost everywhere on $(0, L)$. Recalling that $(2.4 \mathrm{~b})$ holds, let $\xi$ be the infimum of values $x$ such that $\int_{0}^{x} g(s) d s>0$. Evidently $\xi<L$, and furthermore $\xi>0$, otherwise (4.21) would imply

$$
\Phi^{\prime}(s)=0
$$

almost everywhere on $(0, L)$, contradicting (2.17). It is obvious that $g(s)=0$ almost everywhere on $(0, \xi)$. We now show that (4.22) holds for $s>\xi$ : this will complete the proof that $\{\Phi, g\}$ satisfies Condition A. Since

$$
\int_{0}^{s} \beta^{\prime}(W(u, \sigma)) g(u) d u=\beta^{\prime}(W(\bar{u}, \sigma)) \int_{0}^{s} g(u) d u
$$

for some $\bar{u} \in(0, s)$ by the Mean Value Theorem, it follows that

$$
\int_{0}^{s} \beta^{\prime}(W(u, \sigma)) g(u) d u>0, \quad s>\xi .
$$

Then (4.21) implies (4.22) for $s>\xi$, as required.

TheOREM 4. Let $\alpha:[0, \infty) \rightarrow \mathbf{R}$ be continuous, with $\alpha(0)=0$ and $\alpha>0$ on $(0, \infty)$; and let $\alpha^{\prime}(0+)$ exist and be positive. For each $P_{0} \in(0, \infty)$ define $P(L)$ via (2.15) and then $\lambda$ via (2.12). Let $\bar{\lambda}$ denote the supremum (possibly equal to $+\infty$ ) of the set of $\lambda$-values so defined; and let $\beta$ be continuously differentiable on $[0, \bar{\lambda})[$ or $[0, \bar{\lambda}]$ if $\lambda=\bar{\lambda}$ for some $\left.P_{0}\right]$ with $\beta \geqslant 0$ and $\beta^{\prime}>0$ there. Consider pairs $\{f, g\}$ of integrable functions satisfying $(2.1,2.4)$, and for each such pair, define $P$ and $M$ on $[0, L]$ via (2.13a) and (2.13b), for each $P_{0} \in(0, \infty)$. Then the minimum value of $M(L)$ is obtained for each and every $P_{0} \in(0, \infty)$ if and only if $\{f, g\}$ satisfies

Condition A'. There exists a $\xi \in(0, L)$ such that

$$
f(x)=0 \text { a.e., } \quad x>\xi ; \quad g(x)=0 \text { a.e., } x<\xi .
$$

Proof. Recall that, given $\{f, g\}$ and $P_{0}$, and defining $\lambda$ via (2.12), we can define $\Phi$ on $[0, L]$ via (2.16). Then $\Phi$ is absolutely continuous and non-decreasing, and satisfies (2.17). Moreover, $\{\Phi, g\}$ satisfies Condition A of Theorem 3 if and only if $\{f, g\}$ satisfies Condition $\mathrm{A}^{\prime}$, as can be seen from (2.14). We apply Theorem 3 for each $P_{0} \in(0, \infty)$ (and, correspondingly, for each $\lambda$ in $(0, \bar{\lambda})$ or $(0, \bar{\lambda}]$, whichever is appropriate) in order to complete the proof. 
We could examine the extension of this result to the cases where $\beta^{\prime}$ is nowhere negative, but vanishes at some points in $[0, \bar{\lambda}]$. For any given value of $P_{0}$, any pair $\{f, g\}$ satisfying Condition $\mathrm{A}^{\prime}$ (or equivalently, any pair $\{\Phi, g\}$ satisfying Condition A) will then still give rise to the least possible value of $M(L)$ (see Theorem 3 ), but there may now exist pairs not satisfying that condition, which also produce the minimum value. We believe that such other pairs can only be minimising pairs for particular values of $P_{0}$; only pairs satisfying Condition $\mathrm{A}^{\prime}$ produce the minimum $M(L)$ for all $P_{0} \in(0, \infty)$. However, we shall not attempt a proof here.

[Our analysis has concentrated on the values of substrate concentrations at input to and output from a single sinusoid. If the form of the function $\alpha$, and likewise of $\beta$, varies amongst the many sinusoids of the liver, the sinusoidal outfluxes must be summed before comparisons can be made with the results of sampling from the liver vein. This complication $[1,5]$ does not affect the nature of the result we have obtained here, so long as $\alpha$ and $\beta$ for each sinusoid satisfy the general requirements of Theorem 4. If the output concentration of metabolite is minimised for each sinusoid, it is minimised for the liver as a whole.]

The problem of maximising rather than minimising $M(L)$ could conceivably be of relevance for other enzyme types and other substrates in the blood. In contrast to the minimisation problem, the maximisation problem is easily solved whatever the precise form of $\alpha$ and $\beta$. For given $P_{0}$ and corresponding $\lambda,(2.18)$ shows that the largest possible value of $M(L)$ equals $\Phi(L)(=\lambda)$, since $g$ and $\beta$ are non-negative. However, if $\{f, g\}$ satisfies

Condirion B: There exists a $\xi \in(0, L)$ such that

$$
f(x)=0 \text { a.e., } x<\xi ; \quad g(x)=0 \text { a.e., } x>\xi,
$$

then (2.14) and (2.16) show that $\Phi=0$ on $[0, \xi]$. Then, under reasonable assumptions about $\beta,(2.18)$ implies $M=0$ on $[0, \xi]$. (Recall that $\beta(0)=0$.) But then, using (4.26), we have $M=\Phi$ on $(\xi, L]$ so that in particular, $M(L)=\lambda$, the maximum value. Furthermore, it is not hard to see, again with reasonable assumptions about $\beta$, that any $\{f, g\}$ not satisfying Condition B cannot lead to the maximum $M(L)$.

[The interpretation of this result is clear. The outflux concentration of metabolite is maximised if all metabolite is created from the precursor downstream of the conjugating enzymes, so that none has any chance of being conjugated. The outflux concentration $\hat{M}(L)$ of conjugate would be zero in these circumstances.]

\section{Some unsolved problems}

The results of Theorems 3 and 4 hinge on $\beta$ being non-decreasing at all values of $M$ which arise within the sinusoid, for various values of $P_{0}$. For some forms of $\beta(M)$, that need not be the case, as we have already indicated. The simplest 
realistic counterexample occurs when $\beta(M)$ is reduced at very high metabolite concentrations $M$ by substrate inhibition of the enzyme [7], giving rise to $\beta$ as in (2.8b).

For this particular $\beta$, we have

$$
\beta^{\prime}(M)=m\left(1-M^{2} / K_{1} K_{2}\right) /\left(1+M / K_{1}+M^{2} / K_{1} K_{2}\right)^{2},
$$

so that

$$
\begin{array}{ll}
\beta^{\prime}(M)>0, & M<\sqrt{K_{1} K_{2}}, \\
\beta^{\prime}(M)<0, & M>\sqrt{K_{1} K_{2}} .
\end{array}
$$

If $P_{0}<\sqrt{K_{1} K_{2}}$, then certainly $M<\sqrt{K_{1} K_{2}}$ throughout the sinusoid because of (2.11b), since $\lambda<P_{0}$. It then follows from Theorem 3 that whenever $P_{0}<\sqrt{K_{1} K_{2}}$, the minimum $M(L)$ is obtained if and only if $\{f, g\}$ satisfies Condition $\mathrm{A}^{\prime}$ of Theorem 4 (so that the corresponding $\{\Phi, g\}$ satisfies Condition A of Theorem 3 ).

However, we shall now show that for this $\beta$, such a condition on $\{f, g\}$ (or $\{\Phi, g\})$ is not sufficient to produce the minimum $M(L)$ for all values of $P_{0}$, if $\alpha$ is such that $\lambda$ can become sufficiently large (for example, if $\alpha(P)=p P$ ).

For $\lambda>0$, consider a fixed pair $\{\Phi, g\}$ satisfying the usual conditions, and in addition $g=0$ outside $[L / 3,2 L / 3]$, and $\Phi=\lambda$ on $[L / 3, L]$. (Then Condition A is satisfied with $\xi=L / 3$.) We shall construct a modified pair $\{\hat{\Phi}, g\}[c f .(5.18)]$ for comparison. Choose an integrable function $\psi$ on $[0, L]$, with

(i) $\psi=0$ on $[L / 3,2 L / 3]$;

(ii) $\int_{0}^{L / 3} \psi(x) d x=-k, \int_{2 L / 3}^{L} \psi(x) d x=k$;

where $k$ is a constant with $0<k<\lambda$;

(iii) $\psi \leqslant 0$, and $\Phi(x)+\int_{0}^{x} \psi(s) d s$ non-decreasing on $[0, L / 3]$;

(iv) $\psi \geqslant 0$ on $[2 L / 3, L]$.

(For example, with $0<\gamma<1$ and $k=\gamma \lambda$, set $\psi=-\gamma \Phi^{\prime}$ on $[0, L / 3]$; $\psi=0$ on $[L / 3,2 L / 3]$; and $\psi=3 \gamma \lambda / L$ on $[2 L / 3, L]$.)

Now consider, for $(x, \sigma) \in S=[0, L] \times[0,1]$, the integral equation

$$
W(x, \sigma)=\Phi(x)+\sigma \int_{0}^{x} \psi(s) d s-\int_{0}^{x} \beta(W(s, \sigma)) g(s) d s .
$$

By Theorems 1 and 2, this has a continuous solution satisfying (4.4), and moreover $\partial W / \partial \sigma$ exists and equals $W_{1}(x, \sigma)$, where

$$
W_{1}(x, \sigma)=\int_{0}^{x} \psi(s) d s-\int_{0}^{x} \beta^{\prime}(W(s, \sigma)) g(s) W_{1}(s, \sigma) d s .
$$

This is linear in $W_{1}$, and gives

$$
\begin{aligned}
W_{1}(x, \sigma) \exp \left(\int_{0}^{x} \beta^{\prime}(W(s, \sigma)) g(s) d s\right) \\
=\int_{0}^{x} \psi(s)\left[\exp \left(\int_{0}^{s} \beta^{\prime}(W(u, \sigma)) g(u) d u\right)\right] d s
\end{aligned}
$$


so that

$$
\begin{aligned}
& W_{1}(L, \sigma) \exp \left(\int_{0}^{L} \beta^{\prime}(W(s, \sigma)) g(s) d s\right) \\
& =-k+\int_{2 L / 3}^{L} \psi(s)\left[\exp \left(\int_{L / 3}^{2 L / 3} \beta^{\prime}(W(u, \sigma)) g(u) d u\right)\right] d s
\end{aligned}
$$

We note next that

$$
\begin{aligned}
& W(L / 3, \sigma)=\lambda-\sigma k \\
& W(x, \sigma)=\lambda-\sigma k-\int_{L / 3}^{x} \beta(W(s, \sigma)) g(s) d s, \quad L / 3 \leqslant x \leqslant 2 L / 3, \\
& \geqslant \lambda-k-B
\end{aligned}
$$

where

$$
B=\sup \{\beta(W) \mid W \in[0, \lambda]\} .
$$

(For $\beta$ as in (2.8b), it is clear that $B \leqslant m K_{1} \sqrt{K_{2}} /\left(2 \sqrt{K_{1}}+\sqrt{K_{2}}\right)$, the maximum of $\beta$ on $[0, \infty)$.)

Suppose now that $\alpha$ is such that $\lambda$ can be attained with

$$
\frac{1}{2} \lambda>\max \left(B, \sqrt{K_{1} K_{2}}\right) .
$$

Then (5.8) implies that we can choose $k$ such that

$$
W(x, \sigma) \geqslant \frac{1}{2} \lambda, \quad L / 3 \leqslant x \leqslant 2 L / 3,
$$

and it follows from (5.2b) and (5.10) that

$$
\beta^{\prime}(W(x, \sigma))<0, \quad L / 3 \leqslant x \leqslant 2 L / 3 .
$$

Then, in (5.6),

$$
\begin{gathered}
\exp \left(\int_{L / 3}^{2 L / 3} \beta^{\prime}(W(u, \sigma)) g(u) d u\right)<1, \\
\int_{2 L / 3}^{L} \psi(s)\left[\int_{L / 3}^{2 L / 3} \beta^{\prime}(W(u, \sigma)) g(u) d u\right] d s<k,
\end{gathered}
$$

and so

$$
W_{1}(L, \sigma)<0
$$

Thus

$$
\frac{\partial W(L, \sigma)}{\partial \sigma}<0, \quad \sigma \in(0,1)
$$

so that

$$
W(L, 1)<W(L, 0) .
$$


Now $W(L, 0)$ equals the value of $M(L)$ obtained with the pair $\{\Phi, g\}$, because (5.3) reduces to $(2.18)$ when $\sigma=0$. In the same way, $W(L, 1)$ equals the value of $M(L)$ obtained with the pair $\{\Phi, g\}$ replaced in $(2.18)$ by $\{\hat{\Phi}, g\}$, where

$$
\hat{\Phi}(x)=\Phi(x)+\int_{0}^{x} \psi(s) d s,
$$

and this value of $M(L)$ is smaller than the former one, according to (5.17). Now $\hat{\Phi}$ is, like $\Phi$, absolutely continuous and non-decreasing on $[0, L]$, and satisfies

$$
\hat{\Phi}(0)=0, \quad \hat{\Phi}(L)=\lambda,
$$

so $\{\hat{\boldsymbol{\Phi}}, g\}$ is an allowable candidate as a minimising pair for $M(L)$. Since $\{\hat{\boldsymbol{\Phi}}, g\}$ produces a smaller value of $M(L)$ than $\{\Phi, g\}$, it follows that $\{\Phi, g\}$ cannot be a minimising pair, even though it satisfies Condition A. [Thus, when some of the metabolising enzyme is transferred to a location downstream of the conjugation, and correspondingly, $\Phi$ is changed to $\hat{\Phi}$, some metabolite loses its chance of conjugation; but the effect of that on $M(L)$ is outweighed by the effect of reducing the high metabolite concentration inhibiting the conjugation.]

It is reasonably clear that this argument could be adapted to provide a similar result for more general functions $\beta$ than $(2.8 \mathrm{~b})$, so long as $\beta^{\prime}$ is continuous and negative on an interval $\left[M_{1}, M_{2}\right]$, and $\alpha$ and $P_{0}$ are such that values $M$ in this interval are attained within the interval $0 \leqslant x \leqslant L$. To produce the minimum $M(L)$ in such cases, it is not sufficient that $\{\Phi, g\}$ satisfies Condition $\mathrm{A}$, or equivalently, that $\{f, g\}$ satisfies Condition $\mathrm{A}^{\prime}$.

The question arises whether in such cases minimising pairs $\{f, g\}$ exist at all; and if they do, how they can be determined. We have no answer to the second question, and only a partial answer to the first: in Appendix $C$, we show that minimising pairs $\{\Phi, g\}$, and hence $\{f, g\}$, do exist for all $\beta$ as in Theorem 1 , under the additional assumptions that all candidates $\{\Phi, g\}$ satisfy

$$
\int_{0}^{L} g(x)^{2} d x \leqslant H, \quad \int_{0}^{L} \Phi^{\prime}(x)^{2} d x \leqslant H,
$$

for some (arbitrary) constant $H$. These assumptions are unnatural in the biological context of the problem, and it would be preferable if they could be removed. (We remark that it is possible to replace the second powers in (5.20) by $p$ th powers, for any $p>1$, and still prove existence, but such conditions remain unnatural.)

Supposing minimising pairs do exist for each $P_{0} \in(0, \infty)$, do any exist which are minimising for every such $P_{0}$ ? For the $\beta$ of $(2.8 \mathrm{~b})$, it is now clear from the foregoing that if such pair(s) do exist, they must satisfy Condition $\mathrm{A}^{\prime}$, but that this alone is not sufficient. We conjecture that in these cases such "universal" 
minimising pairs do not exist. Rather we expect that, for values of $P_{0}$ leading to sufficiently large values of $\lambda$, minimising pairs $\{f, g\}$ do not satisfy Condition $\mathrm{A}^{\prime}$ -in short, that for such values of $P_{0}$, minimising density functions $f$ and $g$ must have overlapping supports.

If that be so, another, deeper question arises: Supposing that the liver is presented with a wide variety of input precursor concentrations $P_{0}$; that it is desirable to make small the output metabolite concentration $M(L)$; that the kinetic functions $\alpha(P)$ and $\beta(M)$ are such that no distribution of the enzymes, described by $\{f, g\}$, minimises $M(L)$ for all relevant values of $P_{0}$; and that one choice of $\{f, g\}$ has been made; which choice should Nature have made?

Whether or not this question leads to a sensible mathematical problem may depend in particular on the form of the kinetic function $\alpha$, associated with the metabolisation kinetics. If $\alpha$ is such that, as $P_{0}$ runs over $(0, \infty)$, the set of $\lambda$ values obtained is bounded above, then by $(2.11 \mathrm{~b})$, the set of possible values of $M(L)$ is also bounded above. Perhaps in such cases we should look for pairs $\{f, g\}$ which minimise

$$
\sup \left\{M(L) \mid P_{0} \in(0, \infty)\right\}
$$

We hope to return to some of these considerations.

\section{Concluding remarks}

It has often been noted that while variational formulations of the laws of inanimate nature look purposive, they are equivalent to differential formulations that can be viewed causally: the laws themselves are "indifferent with respect to causality and finality" [11]. By contrast, variational principles in biology may express purposiveness in a real sense based on natural selection. Thus, the case for the foregoing minimisation has been argued as follows [4]: "It is tempting to speculate about the advantage (in the sense of natural selection) of zonal arrangement of liver function. The variety of substances which can be synthesized and modified by the liver would be severely restricted if no by-products toxic to the brain and other organs were to occur. This obstacle to evolution would be removed by placing all potentially deleterious hepatic production in a zone $[f(x) \neq 0]$ located upstream of a detoxifying zone $[g(x) \neq 0]$.

Such arguments may be advanced for systems sufficiently limited in space and time (organs, individuals, species). That the indifference with respect to causality and finality would be restored on a larger canvas including the history of unsuccessful mutants, is the essence of the modern theory of evolution. 


\section{Appendix A. Proof of Theorem 1}

We begin with the proof of uniqueness; during this, $\sigma$ is a fixed number in $[0,1]$. Let $\Lambda$ be the Lipschitz constant of $\beta$. We observe firstly that every solution $W(\cdot, \sigma)$ of $(4.3)$ is bounded on $[0, L]$, so that if $W_{A}$ and $W_{B}$ are any two solutions, we have

$$
\left|W_{A, B}(x, \sigma)\right| \leqslant K, \quad x \in[0, L]
$$

for some constant $K$. Put $E(x)=\left|W_{A}(x, \sigma)-W_{B}(x, \sigma)\right|$. Then we have from (4.3)

$$
E(x) \leqslant \Lambda \int_{0}^{x}|g(s)| E(s) d s
$$

It follows from Gronwall's Lemma [9] that $E(x)=0$ for every $x \in[0, L]$, but a direct proof is also easily given. We have from (A.1) and (A.2),

$$
E(x) \leqslant 2 K \Lambda \int_{0}^{x}|g(s)| d s=2 K \Lambda G(x), \text { say, }
$$

and then, using (A.2) repeatedly,

$$
\begin{gathered}
E(x) \leqslant \Lambda \int_{0}^{x} 2 K \Lambda G(s)|g(s)| d s=2 K \Lambda^{2} G(x)^{2} / 2 \\
E(x) \leqslant \Lambda \int_{0}^{x} 2 K \Lambda^{2}\left(G(s)^{2} / 2\right)|g(s)| d s=2 K \Lambda^{3} G(x)^{3} / 3 ! \\
\vdots \\
E(x) \leqslant 2 K \Lambda^{n} G(x)^{n} / n !
\end{gathered}
$$

for every integer $n$. Letting $n \rightarrow \infty$, we obtain $E(x)=0$, so that there are not two distinct solutions $W_{A}, W_{B}$ of (4.3).

We turn now to the question of existence of solutions. If (ivb) holds, we extend $\beta$ by setting $\beta(M)=\beta(0)$ for $M<0$. This extended $\beta$ remains Lipschitz with the same constant $\Lambda$. Define $w_{1}(x, \sigma)=\theta(x, \sigma)$ and, for $n=1,2, \ldots$,

$$
w_{n+1}(x, \sigma)=\theta(x, \sigma)-\int_{0}^{x} g(s) \beta\left(w_{n}(s, \sigma)\right) d s .
$$

We note that $w_{1}(x, \sigma) \leqslant \lambda$, and hence, using (A.5), that if (ivb) holds, then $w_{n}(x, \sigma) \leqslant \lambda$ for all $n \in \mathbf{N}$ and all $(x, \sigma) \in S$. 
Now

$$
\begin{aligned}
&\left|w_{2}(x, \sigma)-w_{1}(x, \sigma)\right| \leqslant \int_{0}^{x}|g(s)||\beta(\Theta(s, \sigma))| d s=C_{1}, \\
&\left|w_{3}(x, \sigma)-w_{2}(x, \sigma)\right| \leqslant \int_{0}^{x} \Lambda C_{1}|g(s)| d s=\Lambda C_{1} G(x), \\
&\left|w_{4}(x, \sigma)-w_{3}(x, \sigma)\right| \leqslant \int_{0}^{x} \Lambda^{2} C_{1} G(s)|g(s)| d s=C_{1}[\Lambda G(x)]^{2} / 2, \\
& \vdots \\
&\left|w_{n+1}(x, \sigma)-w_{n}(x, \sigma)\right| \leqslant C_{1}[\Lambda G(x)]^{n-1} /(n-1) ! \\
& \leqslant C_{1}[\Lambda G(L)]^{n-1} /(n-1) !
\end{aligned}
$$

This last inequality proves uniform convergence of $\left\{w_{n}\right\}$ on $S$. Since each $w_{n}$ is continuous on $S$, so is the limit $W$. By continuity of $\beta$ we have

$$
\beta\left(w_{n}(x, \sigma)\right) \rightarrow \beta(W(x, \sigma)) .
$$

Using the identity

$$
w_{n+1}=w_{1}+\left(w_{2}-w_{1}\right)+\cdots+\left(w_{n+1}-w_{n}\right)
$$

we obtain with the help of (A.6)

$$
\left|w_{n+1}\right| \leqslant \lambda+C_{1} \exp (\Lambda G(L))=C_{2} .
$$

On $\left[-C_{2}, C_{2}\right]$ the function $\beta$ is bounded, and the dominated convergence theorem then implies

$$
\int_{0}^{x} g(s) \beta\left(w_{n}(x, \sigma)\right) d s \rightarrow \int_{0}^{x} g(s) \beta(W(s, \sigma)) d s .
$$

It follows that $W$ is the solution of (4.3).

If (ivb) holds we have $w_{n} \leqslant \lambda$ and hence also $W \leqslant \lambda$. It remains to show that $W(x, \sigma) \geqslant 0$ on $S$. Suppose to the contrary that $W\left(x_{1}, \bar{\sigma}\right)<0$ for some $\left(x_{1}, \bar{\sigma}\right) \in$ $S$; then let

$$
x_{0}=\inf \left\{x ; W(t, \bar{\sigma})<0 \text { for } x \leqslant t \leqslant x_{1}\right\} .
$$

Clearly $0 \leqslant x_{0}<x_{1}, W\left(x_{0}, \bar{\sigma}\right)=0$, and $W(x, \bar{\sigma})<0$ on $\left(x_{0}, x_{1}\right]$. Now we have

$$
\begin{aligned}
& W\left(x_{1}, \bar{\sigma}\right)=W\left(x_{1}, \bar{\sigma}\right)-W\left(x_{0}, \bar{\sigma}\right) \\
& \quad=\theta\left(x_{1}, \bar{\sigma}\right)-\theta\left(x_{0}, \bar{\sigma}\right)-\int_{0}^{x_{1}} \beta(W(s, \bar{\sigma})) g(s) d s+\int_{0}^{x_{0}} \beta(W(s, \bar{\sigma})) g(s) d s \\
& =\theta\left(x_{1}, \bar{\sigma}\right)-\theta\left(x_{0}, \bar{\sigma}\right) \geqslant 0,
\end{aligned}
$$

a contradiction. The proof is complete. 


\section{Appendix B. Proof of Theorem 2}

Let $\varepsilon>0$. It follows from Theorem 1 that (4.6) has a unique, continuous solution $W_{1}$. This $W_{1}$ is necessarily bounded, by $C$ say. We define

$$
E(x)=\left|\frac{W(x, \sigma+h)-W(x, \sigma)}{h}-W_{1}(x, \sigma)\right|
$$

for $h \neq 0$. Then

$$
\begin{aligned}
E(x) \leqslant & \left|\frac{\theta(x, \sigma+h)-\theta(x, \sigma)}{h}-\frac{\partial \theta(x, \sigma)}{\partial \sigma}\right| \\
& +\int_{0}^{x} g(s)\left|\frac{\beta(W(s, \sigma+h))-\beta(W(s, \sigma))}{h}-\beta^{\prime}(W(s, \sigma)) W_{1}(s, \sigma)\right| d s .
\end{aligned}
$$

By the Mean Value Theorem there exists $\gamma, 0<\gamma<1$, such that

$$
\theta(x, \sigma+h)-\theta(x, \sigma)=\frac{\partial \theta(x, \sigma+\gamma h)}{\partial \sigma} h ;
$$

by uniform continuity of $\partial \theta / \partial \sigma$ there exists a positive $\delta_{1}$ such that the first absolute value on the right hand side of (B.2) is smaller than $\varepsilon$ whenever $|h|<\delta_{1}$.

We write

$$
\Delta W=W(x, \sigma+h)-W(x, \sigma)
$$

and using the Mean Value Theorem again, we find $\tau, 0<\tau<1$, such that

$$
\beta(W(x, \sigma+h))-\beta(W(x, \sigma))=\beta^{\prime}(W(x, \sigma)+\tau \Delta W) \Delta W=\beta^{\prime}(\tilde{W}) \Delta W,
$$

say. It follows from (B.2) that

$$
E(x) \leqslant \varepsilon+\int_{0}^{x} g(s)\left|\beta^{\prime}(\tilde{W})\right| E(s) d s+\int_{0}^{x} g(s)\left|\beta^{\prime}(\tilde{W})-\beta^{\prime}(W)\right|\left|W_{1}(s, \sigma)\right| d s
$$

for $|h|<\delta_{1}$.

Since $\tilde{W}$ lies between $W(x, \sigma)$ and $W(x, \sigma+h)$ it follows that $0 \leqslant \tilde{W} \leqslant \lambda$. The continuous function $\beta^{\prime}$ is bounded on $[0, \lambda]$, say by $B_{1}$, and we obtain from (B.6)

$$
E(x) \leqslant \varepsilon+B_{1} \int_{0}^{x} g(s) E(s) d s+C \int_{0}^{x} g(s)\left|\beta^{\prime}(\tilde{W})-\beta^{\prime}(W)\right| d s .
$$

Using uniform continuity of $\beta^{\prime}$ we find a positive $\eta$ such that $\left|\beta^{\prime}(u)-\beta^{\prime}(v)\right|<\varepsilon$ whenever $|u-v|<\eta$, and $u, v \in[0, \lambda]$.

By Theorem $1, W$ is continuous, and therefore there exists a positive $\delta_{2}$ such that, whenever $|h|<\delta_{2}$,

$$
|\Delta W|=|W(x, \sigma+h)-W(x, \sigma)|<\eta .
$$


Hence it follows that $\left|\beta^{\prime}(\tilde{W})-\beta^{\prime}(W)\right|<\varepsilon$ if $|h|<\delta_{2}$. Then we have from (B.7) that, for $|h|<\min \left(\delta_{1}, \delta_{2}\right)$,

$$
E(x) \leqslant \varepsilon+\varepsilon C G(L)+B_{1} \int_{0}^{x} g(s) E(s) d s,
$$

with $G(x)$ as in (A.3). Using Gronwall's Lemma [9] we obtain

$$
E(x) \leqslant \varepsilon(1+C G(L)) \exp (B, G(L)) .
$$

From this inequality, and the definition (B.1) of $E(x)$, we can see that $\partial W / \partial \sigma$ exists and equals $W_{1}$.

\section{Appendix C. Existence of minimising pairs}

For given $\lambda>0$, and given $\beta$ Lipschitz and non-negative on $[0, \lambda]$, we consider (2.18) for various pairs $\{\Phi, g\}$; with $\Phi$ absolutely continuous and non-decreasing on $[0, L]$, and satisfying (2.17); and $g$ integrable on $[0, L]$ and satisfying (2.4). For each such $\{\Phi, g\}$, we know that (2.18) has a unique, continuous solution $M$ satisfying $(2.1 \mathrm{~b})$, so that, in particular,

$$
0 \leqslant M(L) \leqslant \lambda \text {. }
$$

Let $\phi=\Phi^{\prime}$; then $\phi \geqslant 0$ a.e. and $\phi$ integrable on $[0, L]$, with

$$
\int_{0}^{L} \phi(x) d x=\lambda \text {. }
$$

Suppose now that we allow only those $\{\Phi, g\}$ or, correspondingly, $\{\phi, g\}$, which satisfy the additional conditions(5.20) for some constant $H$. We set equal to $a$ the infimum of the $M(L)$ values obtained from (2.18) with all such pairs.

There exists a sequence of such pairs $\left\{\phi_{n}, g_{n}\right\}$ such that for the corresponding solutions $M_{n}(x)$ of $(2.18)$ we have

$$
M_{n}(L) \rightarrow a .
$$

Given (5.20) we can select a subsequence $\left\{\phi_{n}, g_{n}\right\}$ (we do not change the subscripts when passing to a subsequence) such that $\phi_{n}$ and $g_{n}$ are weakly convergent,

$$
\phi_{n} \rightarrow \phi, \quad g_{n} \rightarrow g \quad \text { in } L_{2}(0, L) .
$$

The corresponding $M_{n}(x)$ are continuous and bounded, and

$$
\begin{aligned}
\left|M_{n}\left(x_{2}\right)-M_{n}\left(x_{1}\right)\right| & \leqslant \int_{x_{1}}^{x_{2}} \phi_{n}(x) d x+\int_{x_{1}}^{x_{2}} g_{n}(x) \beta\left(M_{n}(x)\right) d x \\
& \leqslant \sqrt{x_{2}-x_{1}} \int_{0}^{L} \phi_{n}(x)^{2} d x+B \sqrt{x_{2}-x_{1}} \int_{0}^{L} g_{n}(x)^{2} d x,
\end{aligned}
$$


where $B$ is a bound for $\beta$ on $[0, \lambda]$. Thus

$$
\left|M_{n}\left(x_{2}\right)-M_{n}\left(x_{1}\right)\right| \leqslant(1+B) H \sqrt{x_{2}-x_{1}} .
$$

Clearly the $M_{n}(x)$ are equicontinuous; and there exists a uniformly convergent subsequence $M_{n}(x) \rightarrow \bar{M}(x)$, say. Since $\beta$ is Lipschitz, the sequence $\beta\left(M_{n}(x)\right.$ ) then also converges uniformly, and therefore converges in $L_{2}(0, L)$.

Let $h$ denote the characteristic function of $[0, x]$, and $(\cdot, \cdot),\|\cdot\|$ the usual scalar product and norm for $L_{2}(0, L)$. We have

$$
\int_{0}^{x} \phi_{n}(s) d s=\left(\phi_{n}, h\right) \rightarrow(\phi, h)=\int_{0}^{x} \phi(s) d s
$$

and

$$
\begin{aligned}
\int_{0}^{x} g_{n}(s) \beta\left(M_{n}(s)\right) d s & =\left(g_{n}, \beta\left(M_{n}\right) h\right) \\
& =\left(g_{n}, \beta(\widehat{M}) h\right)+\left(g_{n},\left[\beta\left(M_{n}\right)-\beta(\bar{M})\right] h\right) .
\end{aligned}
$$

Now

$$
\begin{aligned}
\left(g_{n}, \beta(\bar{M}) h\right) \rightarrow(g, \beta(\bar{M}) h) & =\int_{0}^{x} g(s) \beta(\bar{M}(s)) d s, \\
\left(g_{n},\left[\beta\left(M_{n}\right)-\beta(\bar{M})\right] h\right) & \leqslant\left\|g_{n}\right\|\left\|\beta\left(M_{n}\right)-\beta(\bar{M})\right\| \\
& \leqslant \sqrt{H}\left\|\beta\left(M_{n}\right)-\beta(\bar{M})\right\| \\
& \rightarrow 0 .
\end{aligned}
$$

Therefore we have

$$
\int_{0}^{x} g_{n}(s) \beta\left(M_{n}(s)\right) d s \rightarrow \int_{0}^{x} g(s) \beta(\bar{M}(s)) d s .
$$

By sending $n \rightarrow \infty$ in

$$
M_{n}(x)=\int_{0}^{x}\left[\phi_{n}(s)-g_{n}(s) \beta\left(M_{n}(s)\right)\right] d s
$$

we get

$$
\bar{M}(x)=\int_{0}^{x}[\phi(s)-g(s) \beta(\bar{M}(s))] d s .
$$

Thus $\bar{M}(x)$ is a solution of $(2.18)$ with the pair $\{\Phi, g\}$, and moreover $\bar{M}(L)=$ $\lim M_{n}(L)=a$. We have proved the existence of a minimising pair $\{\Phi, g\}$.

\section{References}

[1] L. Bass, P. J. Robinson and A. J. Bracken, "Hepatic elimination of flowing substrates: the distributed model", J. Theoret. Biol. 72 (1978), 161-184.

[2] L. Bass, "On the location of cellular functions in perfused organs", J. Theoret. Biol. 82 (1980), 347-351. 
[3] L. Bass, "Functional zones in rat liver: the degree of overlap", J. Theoret. Biol. 89 (1981), 303-319.

[4] L. Bass, "Functional zones in the liver", Gastroenterology 81 (1981), 976-977.

[S] A. J. Bracken and L. Bass, "Statistical mechanics of hepatic elimination", Math. Biosci. 44 (1979), $97-120$.

[6] E. A. Coddington and N. Levinson, Theory of ordinary differential equations (McGraw-Hill, New York, 1955), 43.

[7] M. Dixon and E. C. Webb, Enzymes (Longmans, London, 3rd edition, 1979).

[8] J. J. Gumució and D. L. Miller, "Functional implications of liver cell heterogeneity", Gastroenterology 80 (1981), 393-403.

[9] E. Hille, Lectures on differential equations (Addison-Wesley, Reading, Mass., 1969), 19.

[10] K. S. Pang and J. R. Gillette, "Kinetics of metabolite formation and elimination in the perfused rat liver preparation", J. Pharmacol. Exp. Ther. 207 (1978), 178-194.

[11] H. Weyl, Philosophy of mathematics and natural science (Princeton Univ. Press, Princeton, N.J., 1949), Chapter 3. 